\title{
PeCAn: Compositional Verification of Petri Nets Made Easy ${ }^{\star}$
}

\author{
Dinh-Thuan Le ${ }^{1}$, Huu-Vu Nguyen ${ }^{1}$, Van-Tinh Nguyen ${ }^{1}$, Phuong-Nam Mai ${ }^{1}$, \\ Bao-Trung Pham-Duy ${ }^{1}$, Thanh-Tho Quan ${ }^{1}$, Étienne André ${ }^{2}$, Laure Petrucci ${ }^{2}$, \\ Yang $\mathrm{Liu}^{3}$ \\ ${ }^{1}$ HoChiMinh City University of Technology, Vietnam \\ ${ }^{2}$ Université Paris 13, Sorbonne Paris Cité, LIPN, CNRS, Villetaneuse, France \\ ${ }^{3}$ Nanyang Technological University, Singapore
}

\begin{abstract}
This paper introduces PeCAn, a tool supporting compositional verification of Petri nets. Beyond classical features (such as onthe-fly analysis and synchronisation between multiple Petri nets), PeCAn generates Symbolic Observation Graphs (SOG), and uses their composition to support modular abstractions of multiple Petri nets for more efficient verification. Furthermore, PeCAn implements an incremental strategy based on counter-examples for model-checking, thus improving significantly the cost of execution time and memory space. PeCAn also provides users with the visualisation of the input Petri nets and their corresponding SOGs. We experimented PeCAn with benchmark datasets from the Petri Nets' model checking contests, showing promising results.
\end{abstract}

Keywords: Compositional verification, Petri nets, SOG

\section{Introduction}

A Petri net (PN) [KNN02] is a graphical mathematical language which efficiently supports the modelling and verification of distributed systems. Basically, a Petri net is a directed bipartite graph, featuring transitions and places. As Petri nets are widely used in research and industry communities, there are several tools developed to help users specify and verify Petri nets, in particularly LoLa [Sch03], Snoopy [HRS08], TAPAAL [BJS09], CosyVerif [AHHH ${ }^{+}$13], CPN Tools [Wes13] or JPetriNet ${ }^{1}$. Although most of the tools work with basic place/transitions PNs, some of them cater for some advanced forms of PNs such as timed, coloured, or stochastic PNs.

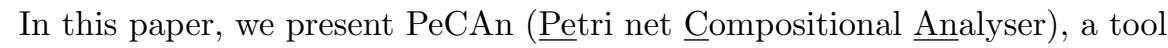
supporting verification of Petri nets in a compositional manner. PeCAn can take

\footnotetext{
* This work is partially supported by the STIC-Asie project CATS ("Compositional Analysis of Timed Systems"). This is the author version of the paper of the same name accepted for publication at the 12th International Symposium on Automated Technology for Verification and Analysis (ATVA 2014). The final version is available at www. springer. com.

${ }^{1}$ http://jpetrinet.sourceforge.net
} 
as input Petri Net models described in PNML, one of the most popular languages to describe Petri Nets nowadays. The properties to be checked are expressed as LTL formulae. PeCAn offers the following features:

- PeCAn allows users to compose a complex PN from multiple concurrent PNs and then verify the composed PN against a given property.

- PeCAn is able to generate Symbolic Observation Graphs (SOG) [HIK04] from the actual PNs. Therefore, PeCAn supports verification of modular PNs by composing SOGs of separate components.

- PeCAn implements the incremental strategy based on counter-examples when verifying the generated SOG [AKOP12]. Thus, the cost of execution time and memory space is significantly reduced.

\section{Modular Verification}

In this section, we take the example presented in [LP10] to demonstrate how to use PeCAn to verify Petri nets. Even though PeCAn can verify a single Petri net as other existing tools do, in this paper we only focus on compositional verification of PeCAn, i.e. verifying a Petri net composed by multiple synchronised modules.

We assume that the original Petri net is already decomposed by users into modules. PeCAn allows users to verify an arbitrary composition of predefined modules. In order to do so, they must define synchronised transitions by the same name between modules. Figure $1 \mathrm{~b}$ gives an example of a system decomposed into three modules through synchronised transitions. This system can be described easily in a modular style by PeCAn. In this example, modules A and B have two transitions with the same name (F1, F3) meaning that these two transitions must be synchronised. Similarly, a synchronised transition, F2, is shared by modules $\mathrm{B}$ and $\mathrm{C}$, also declared by the same name in PeCAn.

When the module composition and the LTL property are defined, users can choose to perform the verification using one of the following methods:

Basic LTL Verification The modules are synchronised together based on the user specification. Then the synchronised modules are converted into an LTS model and verified on-the-fly by the PAT model checking library [SLDP09].

SOG-based Verification In this method, we do not directly verify the synchronised modules. Instead, we produce a corresponding SOG and use it for the verification. If a counter-example is found, it is verified again on the original Petri net to check whether it is an actual counter-example.

Incremental SOG-based Verification It is similar to the SOG-based Verification method. However, we do not generate the SOG for the whole synchronisation of modules. Instead, we incrementally synchronise two modules first and verify the corresponding SOG. If no counter-example is found, we incrementally synchronise one more module and repeat the SOG-based verification step, until a counter-example is found or all modules are synchronised and verified (see [AKOP12]). 


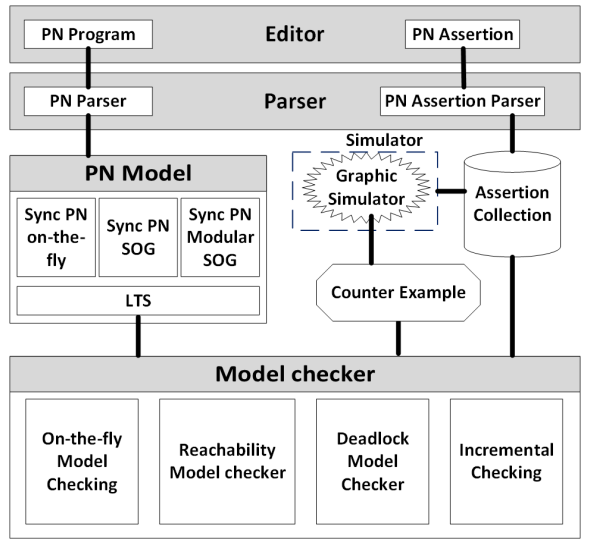

(a) Architecture of PeCAn

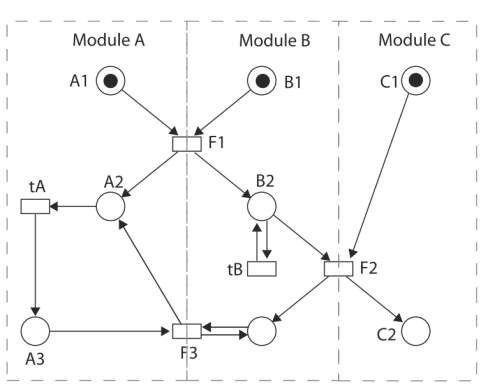

(b) A modular PN example

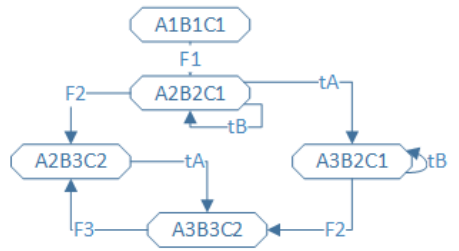

(c) Full state space of the model

Fig. 1: Architecture of PeCAn and example with state space

\section{Architecture}

The architecture of PeCAn, given in Figure 1a, is described as follows:

Editor Layer Allows users to describe PNs by a (i) PNML specification or (ii) graph-based visualisation. Users can design an arbitrary number of modules as well as any composition between them.

Parser Layer Parses the architectures of the PNs from the Editor Layer and converts the Petri net models as well as properties to check into an internal representation for the Semantic Layer.

Semantic Layer Responsible for generating the corresponding LTS of the input Petri nets, in order to be model checked by the next layer. The three approaches of Basic LTL Verification, SOG-based Verification and Incremental SOG-based Verification are then implemented as three sub-modules: Sync PN on-the-fly, Sync PN SOG and Sync PN Modular SOG.

Model checker Layer We make use of the PAT model checking library [SLDP09] for this layer. This library takes an LTS as input, and verifies the properties.

\section{Functionality Comparison and Experiments}

We finally present some comparative discussion and experiments of our tool with other similar approaches. Since PeCAn takes PNML as input, we collected other PN verification tools also supporting PNML. We selected the tools listed at 
http://www . informatik. uni-hamburg. de/TGI/PetriNets/tools/ and supporting PNML. As shown in Table 1, very few tools can support PNML specification and perform full LTL verification.

\begin{tabular}{|c|l|c|c|c|c|c|}
\hline No & \multicolumn{1}{|c|}{ Tool } & $\begin{array}{c}\text { PNML format } \\
\text { supported }\end{array}$ & $\begin{array}{c}\text { GUI } \\
\text { editor }\end{array}$ & $\begin{array}{c}\text { Deadlock } \\
\text { checking }\end{array}$ & $\begin{array}{c}\text { User-defined } \\
\text { LTL checking }\end{array}$ & Simulation \\
\hline 1 & PeCAn & $\checkmark$ & $\checkmark$ & $\checkmark$ & $\checkmark$ & $\checkmark$ \\
\hline 2 & PNEditor $^{2}$ & $\checkmark$ & $\checkmark$ & $\times$ & $\times$ & \\
\hline 3 & Snoopy $^{3}$ & $\times^{4}$ & $\checkmark$ & $\times$ & $\times$ & \multicolumn{2}{|c|}{} \\
\hline 4 & PNML Framework $^{5}$ & $\checkmark$ & $\checkmark$ & \multicolumn{3}{|c|}{} \\
\hline 5 & ProM framework $^{7}$ & $\checkmark$ & $\checkmark$ & $\times$ & $?^{6}$ & $?^{8}$ \\
\hline 6 & P3 $^{9}$ & $\checkmark$ & & $\times$ & & $\checkmark$ \\
\hline 7 & ePNK $^{10}$ & $\checkmark$ & $\checkmark$ & $?^{11}$ & & $\checkmark$ \\
\hline 8 & Tina $^{12}$ & $\checkmark$ & $\times$ & $\times$ & $\checkmark$ & \\
\hline
\end{tabular}

Table 1: Available tools that support PNML models

We then experimented PeCAn with benchmark datasets downloaded from the Model Checking contest $\left[\mathrm{KLB}^{+} 13\right]^{13}$. Results, as display in Table 2 showed that PeCAn can endure some remarkably large model sizes. When a counterexample is found, PeCAn can terminate quickly with significantly less resources usage.

Lastly, we also compared the performance of PeCAn in terms of the (symbolic) states and transitions generated by the SOG-based approach. The results are presented in Figures $2 \mathrm{a}$ and $2 \mathrm{~b}$ respectively. Results show that the SOGbased approach of PeCAn usually reduces the number of states, and always significantly reduces the number of transitions when compared to the standard approach. In fact, the number of generated transitions is always significantly reduced, leading to a substantial gain of time when applying a model checking algorithm. The tool and all experiments can be downloaded from [Le14].

Acknowledgments. All our thanks to the PAT team [SLDP09] for their help in interfacing our tool with the PAT library.

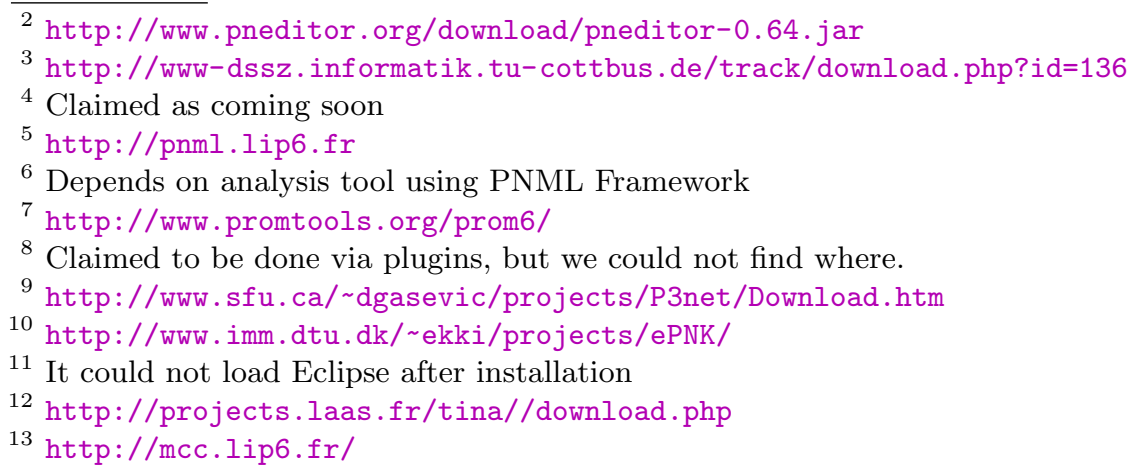




\begin{tabular}{|c|c|c|c|c|c|c|c|}
\hline No & Model & Parameter & State space & $\begin{array}{l}\text { Number of } \\
\text { Reached } \\
\text { Markings }\end{array}$ & $\begin{array}{c}\text { Number of } \\
\text { Transition } \\
\text { Firings }\end{array}$ & $\underset{(\mathrm{s})}{\operatorname{Time}}$ & $\begin{array}{c}\text { Memory } \\
(\mathrm{KB})\end{array}$ \\
\hline \begin{tabular}{|l|}
1 \\
\end{tabular} & CSRepetitions & 2 & 7.424 & 32 & 31 & \begin{tabular}{|l|}
0.015 \\
\end{tabular} & 8,962 \\
\hline 2 & CSRepetitions & 3 & $1.341 \times 10^{8}$ & 117 & 116 & \begin{tabular}{|l|}
0.078 \\
\end{tabular} & 10,465 \\
\hline 3 & CSRepetitions & 4 & unknown & 291 & 290 & 0.202 & 16,033 \\
\hline 4 & CSRepetitions & 5 & unknown & 1,274 & 1,283 & 0.642 & 43,289 \\
\hline 5 & CSRepetitions & 7 & unknown & 2,148 & 2,147 & 2.367 & 135,809 \\
\hline 6 & CSRepetitions & 8 & unknown & 7,242 & 7,241 & 20.836 & $1,056,194$ \\
\hline 7 & Eratosthenes & 10 & 32 & 12 & 19 & \begin{tabular}{|l|}
0.191 \\
\end{tabular} & 8,635 \\
\hline 8 & Eratosthenes & 20 & 2,048 & 28 & 60 & \begin{tabular}{|l|}
0.015 \\
\end{tabular} & 8,929 \\
\hline 9 & Eratosthenes & 50 & $1.718 \times 10^{10}$ & 287 & 821 & \begin{tabular}{|l|}
0.071 \\
\end{tabular} & 11,451 \\
\hline 10 & Eratosthenes & 100 & $1.899 \times 10^{22}$ & 1,236 & 4,099 & 0.539 & 23,446 \\
\hline \begin{tabular}{|l|}
11 \\
\end{tabular} & Eratosthenes & 200 & $1.142 \times 10^{46}$ & 3,614 & 13,007 & \begin{tabular}{|l|}
4.794 \\
\end{tabular} & 91,365 \\
\hline \begin{tabular}{|l|}
12 \\
\end{tabular} & Eratosthenes & 500 & $4.13 \times 10^{121}$ & 24,236 & 88,363 & 76.082 & 899,525 \\
\hline 13 & HouseContruction & 2 & 1,501 & 74 & 73 & \begin{tabular}{|l|}
0.119 \\
\end{tabular} & 9,100 \\
\hline \begin{tabular}{|l|}
14 \\
\end{tabular} & HouseContruction & 5 & unknown & 209 & 208 & \begin{tabular}{|l|}
0.031 \\
\end{tabular} & 10,095 \\
\hline 15 & HouseContruction & 10 & $1,664 \times 10^{9}$ & 434 & 433 & \begin{tabular}{|l|}
0.018 \\
\end{tabular} & 10,354 \\
\hline \begin{tabular}{|l|}
16 \\
\end{tabular} & HouseContruction & 20 & $1.367 \times 10^{13}$ & 884 & 883 & \begin{tabular}{|l|}
0.052 \\
\end{tabular} & 13,481 \\
\hline 17 & HouseContruction & 50 & unknown & 2,234 & 2,233 & 0.121 & 17,747 \\
\hline \begin{tabular}{|l|}
18 \\
\end{tabular} & HouseContruction & 100 & unknown & 4,484 & 4,483 & 0.294 & 20,059 \\
\hline 19 & HouseContruction & 200 & unknown & 8,984 & 8,983 & \begin{tabular}{|l|}
0.471 \\
\end{tabular} & 32,975 \\
\hline 20 & HouseContruction & 500 & unknown & 22,484 & 22,483 & 1.48 & 63,711 \\
\hline 21 & PermAdmissibility & 1 & 52,537 & 41 & 40 & \begin{tabular}{|l|}
0.183 \\
\end{tabular} & 10,437 \\
\hline 22 & PermAdmissibility & 2 & unknown & 253 & 252 & \begin{tabular}{|l|}
0.098 \\
\end{tabular} & 13,243 \\
\hline \begin{tabular}{|l|}
23 \\
\end{tabular} & PermAdmissibility & 5 & unknown & 1,025 & 1,024 & \begin{tabular}{|l|}
0.363 \\
\end{tabular} & 25,011 \\
\hline 24 & PermAdmissibility & 10 & unknown & 2,372 & 2,371 & \begin{tabular}{|l|}
0.869 \\
\end{tabular} & 45,072 \\
\hline 25 & PermAdmissibility & 20 & unknown & 5,027 & 5,026 & 2.021 & 87,138 \\
\hline 26 & PermAdmissibility & 50 & unknown & 12,912 & 12,911 & 4.901 & 201,224 \\
\hline \begin{tabular}{|l|}
27 \\
\end{tabular} & Philosopher & 5 & 243 & 68 & 84 & \begin{tabular}{|l|}
0.007 \\
\end{tabular} & 9,274 \\
\hline 28 & Philosopher & 10 & 59,049 & 7,242 & 10,576 & $\mid 1.057$ & 42,935 \\
\hline 29 & Philosopher & 20 & $3.487 \times 10^{9}$ & \multicolumn{4}{|c|}{ Time out after $7200 \mathrm{~s}$} \\
\hline
\end{tabular}

Table 2: Experiments with deadlock models: PeCAn does not need to explore the whole state space 


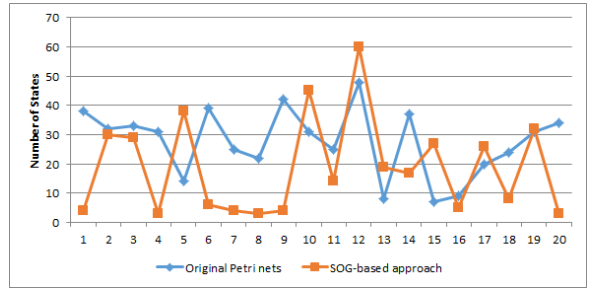

(a) Number of states

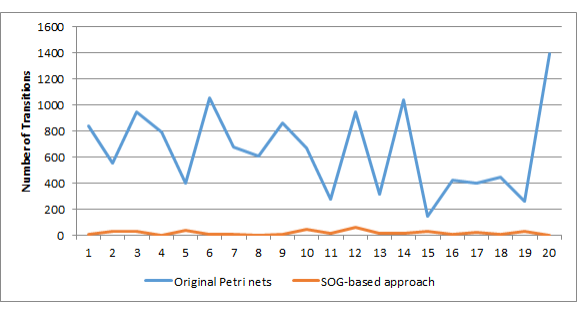

(b) Number of transitions

Fig. 2: Experimental results on a set of Petri nets

\section{References}

$\mathrm{AHHH}^{+}$13. Étienne André, Lom-Messan Hillah, Francis Hulin-Hubard, Fabrice Kordon, Yousra Lembachar, Alban Linard, and Laure Petrucci. CosyVerif: An open source extensible verification environment. In ICECCS, pages 33-36. IEEE Computer Society, 2013.

AKOP12. Étienne André, Kais Klai, Hanen Ochi, and Laure Petrucci. A counterexample-based incremental and modular verification approach. In Monterey Workshop, pages 283-302, 2012.

BJS09. Joakim Byg, Kenneth Yrke Jørgensen, and Jirí Srba. TAPAAL: Editor, simulator and verifier of timed-arc Petri nets. In ATVA, LNCS, pages 84-89. Springer, 2009.

HIK04. Serge Haddad, Jean-Michel Ilié, and Kais Klai. Design and evaluation of a symbolic and abstraction-based model checker. In ATVA, volume 3299 of LNCS, pages 196-210. Springer, 2004.

HRS08. Monika Heiner, Ronny Richter, and Martin Schwarick. Snoopy: A tool to design and animate/simulate graph-based formalisms. In SimuTools, page $15,2008$.

$\mathrm{KLB}^{+}$13. Fabrice Kordon, Alban Linard, Marco Beccuti, Didier Buchs, Lukasz Fronc, Lom-Messan Hillah, Francis Hulin-Hubard, Fabrice Legond-Aubry, Niels Lohmann, Alexis Marechal, Emmanuel Paviot-Adet, Franck Pommereau, César Rodríguez, Christian Rohr, Yann Thierry-Mieg, Harro Wimmel, and Karsten Wolf. Model checking contest @ Petri nets, report on the 2013 edition. CoRR, abs/1309.2485, 2013.

KNN02. V. E. Kozura, V. A. Nepomniaschy, and R. M. Novikov. Verification of distributed systems modelled by high-level Petri nets. In PARELEC, pages 61-66, 2002.

Le14. Dinh-Thuan Le. PeCAn Web page, 2014. http://cse.hcmut.edu.vn/ save/project/pn-ver/start.

LP10. Charles Lakos and Laure Petrucci. Modular state spaces for prioritised Petri nets. In Monterey Workshop, pages 136-156, 2010.

Sch03. Karsten Schmidt. Distributed verification with LoLA. Fund. Inf., 54(23):253-262, 2003.

SLDP09. Jun Sun, Yang Liu, Jin Song Dong, and Jun Pang. PAT: Towards flexible verification under fairness. In $C A V$, volume 5643 of $L N C S$, pages 709-714. Springer, 2009. 
Wes13. Michael Westergaard. CPN Tools 4: Multi-formalism and extensibility. In Petri Nets, volume 7927 of LNCS, pages 400-409. Springer, 2013. 